\title{
The Study of the Frequency Effect of Dynamic Compressive Loading on Primary Articular Chondrocyte Functions Using a Microcell Culture System
}

\author{
Wan-Ying Lin, ${ }^{1}$ Yu-Han Chang, ${ }^{2}$ Hsin-Yao Wang, ${ }^{1}$ Tzu-Chi Yang, ${ }^{3}$ \\ Tzu-Keng Chiu, ${ }^{4}$ Song-Bin Huang, ${ }^{3}$ and Min-Hsien $\mathrm{Wu}^{3}$ \\ ${ }^{1}$ School of Medicine, Chang Gung University, 259 Wen-Hwa 1st Road, Gueishan Township, Taoyuan County 333, Taiwan \\ ${ }^{2}$ Department of Orthopaedic Surgery, Chang Gung Memorial Hospital, Linko, 5 Fusing St., Gueishan Township, \\ Taoyuan County 333, Taiwan \\ ${ }^{3}$ Graduate Institute of Biochemical and Biomedical Engineering, Chang Gung University, 259 Wen-Hwa 1st Road, \\ Gueishan Township, Taoyuan County 333, Taiwan \\ ${ }^{4}$ Graduate Institute of Chemical and Materials Engineering, Chang Gung University, 259 Wen-Hwa 1st Road, \\ Gueishan Township, Taoyuan County 333, Taiwan \\ Correspondence should be addressed to Min-Hsien Wu; mhwu@mail.cgu.edu.tw
}

Received 3 December 2013; Revised 12 March 2014; Accepted 17 March 2014; Published 16 April 2014

Academic Editor: Nihal Engin Vrana

Copyright (C) 2014 Wan-Ying Lin et al. This is an open access article distributed under the Creative Commons Attribution License, which permits unrestricted use, distribution, and reproduction in any medium, provided the original work is properly cited.

\begin{abstract}
Compressive stimulation can modulate articular chondrocyte functions. Nevertheless, the relevant studies are not comprehensive. This is primarily due to the lack of cell culture apparatuses capable of conducting the experiments in a high throughput, precise, and cost-effective manner. To address the issue, we demonstrated the use of a perfusion microcell culture system to investigate the stimulating frequency $(0.5,1.0$, and $2.0 \mathrm{~Hz})$ effect of compressive loading $(20 \%$ and $40 \%$ strain) on the functions of articular chondrocytes. The system mainly integrates the functions of continuous culture medium perfusion and the generation of pneumatically-driven compressive stimulation in a high-throughput micro cell culture system. Results showed that the compressive stimulations explored did not have a significant impact on chondrocyte viability and proliferation. However, the metabolic activity of chondrocytes was significantly affected by the stimulating frequency at the higher compressive strain of $40 \%(2 \mathrm{~Hz}, 40 \% \mathrm{strain})$. Under the two compressive strains studied, the glycosaminoglycans (GAGs) synthesis was upregulated when the stimulating frequency was set at $1 \mathrm{~Hz}$ and $2 \mathrm{~Hz}$. However, the stimulating frequencies explored had no influence on the collagen production. The results of this study provide useful fundamental insights that will be helpful for cartilage tissue engineering and cartilage rehabilitation.
\end{abstract}

\section{Introduction}

Articular cartilage is a tough and resilient connective tissue lying at the end surface of long bones near joints. The biological function of cartilage to provide resistance to mechanical loading is primarily attributed to its abundant extracellular matrix (ECM), mainly consisting of glycosaminoglycans (GAGs) and collagen [1]. The homeostasis of the ECM is maintained by the articular chondrocytes, the only cell species existing in articular cartilage. Upon damage, adult cartilage has a limited ability of self-repairing. To treat cartilage defects, cartilage tissue engineering is generally regarded as a promising method. The common approach to cartilage tissue engineering involves the in vitro cultivation of tissue constructs by using (1) chondrogenic cells, (2) scaffolds capable of providing a three-dimensional (3D) structure for tissue development, and (3) bioreactors capable of providing the suitable extracellular conditions necessary for the cells to regenerate functional cartilaginous tissues [2]. Although proof of principle has been demonstrated [1], few in vitro 
cartilage tissue engineering investigations have generated an appropriate tissue that meets the functional demands placed upon this tissue in vivo.

One of the key technical hurdles to functional cartilage tissue engineering is the lack of fundamental understanding of the link between extracellular conditions and chondrocyte functions. Though several studies have been carried out to find out the relationship between articular chondrocyte and environment, the majority of them have focused on exploring the effect of biochemical factors on cellular functions [3]. Physical factors like mechanical stimulation have been reported to play an important role in modulating chondrocyte physiology and, in turn, cartilage homeostasis [4]. Although some fundamental investigations have been carried out to probe the effect of mechanical stimulation on articular chondrocyte physiology [1,5-11], the relevant studies are, to some extent, not comprehensive. The possible cause behind this is a lack of appropriate experimental tools, by which the effect of mechanical stimulation on chondrocyte function can be quantitatively determined in an efficient, precise, physiologically meaningful, and cost-effective manner.

The effects of mechanical loading on articular chondrocytes are complex. Under physiological mechanical stimulation, the articular chondrocytes in cartilage are subjected to complicated physical events including hydrostatic pressure, tensile stress, shear stress, and compressive stress [11]. Among these events, compressive stimulation plays an important role in modulating the articular chondrocyte functions [1]. Several devices or bioreactors have been developed for investigating the effect of compressive stimulation on articular chondrocyte physiology. These cell or tissue culture apparatuses commonly make use of the direct compression on the chondrocytes-embedded constructs or on cartilage explants due to its similarity to in vivo loading by intraarticular contact [1]. According to the working regimen, the cell or tissue culture devices capable of providing compressive loading to cells can be divided into two categories, namely, static [5] and dynamic systems [1,6-11]. Dynamic compressive stimulation is predominantly adopted because dynamic loading is physiologically meaningful $[1,12]$. Numerous cell or tissue culture devices, capable of generating dynamic compressive stimulation, have been demonstrated to be feasible for providing compressive loading to the cartilage explants, or chondrocytes-embedded scaffold [1,5-10]. Nevertheless, these devices are normally technically demanding, complicated, large, and costly, which could limit the experimental throughput. Moreover, most of these devices adopt static cell culture format $[1,5-7,9,10,13]$, where the culture medium is literally supplied in a manual and batch-wise manner. This could lead to high contamination risk and fluctuating culture condition [14]. The latter could hamper the precise quantification of the link between the mechanical stimulation and articular chondrocyte functions since reports in literature have demonstrated that the articular chondrocytes are fairly sensitive to extracellular environments [15].

While tissue engineering scientists are making progress [16], they might need an appropriate device to aid their research. To address the issue, a microcell culture system capable of providing dynamic compressive loading to $3 \mathrm{D}$ cell culture constructs was proposed in our previous study (Figure 1(a)) [11]. One of its distinctive features is the function of tunable compressive loading generation. The mechanism is based on the pneumatically driven deformation of an elastic polydimethylsiloxane (PDMS) membrane, which in turn exerts compressive loading onto a 3D cell culture construct through a micropillar (Figure 1(a)) [11]. By modulating the frequency and pressure of the input pneumatic conditions, the compressive loading can be generated in a controllable manner. Other technical features of the microcell culture system are (1) low cost, (2) high throughput, (3) low consumption of research resources due to its small scale, (4) maintaining a stable and well-defined culture condition due to the perfusion culture format and miniaturized culture scale [14, 17], (5) efficient and precise sample (e.g., cells) loading, and (6) easy operation. Borrowing from our previous experience on the micro cellculture system, the frequency $(0.5,1.0$, and $2.0 \mathrm{~Hz})$ effect of dynamic compressive loading (strain: 20 and 40\%) on the cell viability and proliferation as well as the metabolic and biosynthetic activities of primary articular chondrocytes were investigated.

\section{Materials and Methods}

2.1. The Microcell Culture System and Experimental Setup. The microcell culture system is composed of 12 individual microbioreactors (D: $7.5 \mathrm{~mm}$; $\mathrm{H}: 6.5 \mathrm{~mm}$ ). At the bottom of each microbioreactor, there is a cylindrical cavity (D: $1 \mathrm{~mm}$; $\mathrm{H}: 2.3 \mathrm{~mm}$; volume: $1.8 \mu \mathrm{L}$ ), which is not only used to accommodate a cell/biomaterial scaffold for $3 \mathrm{D}$ cell culture but also to quantitatively define the volume of such sample loading. The overall sample-loading process was described previously [11].

For high throughput perfusion cell culture purpose, each microbioreactor was designed to perfuse with its own separate medium supply through silicon tubing driven by a multichannel syringe pump (KDS 220, KD Scientific Ltd., USA). In addition, the microcell culture platform was placed on the surface of a transparent indium-tin-oxide- (ITO-) based microheater chip to provide a stable thermal condition of $37 \pm 0.2^{\circ} \mathrm{C}$ for cell culture [18]. To pneumatically drive and control the compressive loading mechanism, 4 via holes were connected with 4 air tubes from a custom-made pneumatic controller. Within the hand-held controller, an air compressor (MDR2-1A/11, Jun-Air Inc., Japan), 4 electromagnetic valves (EMV) (S070M-5BG-32, SMC Inc., Taiwan), and a programmable control circuit were integrated to activate and to control the generation of compressive loading. The overall experimental setup is illustrated in Figure 1(b).

\subsection{The Exertions of Dynamic Compressive Loading: Oper-} ating Conditions and Long-Term Stability. In this study, the operating stimulating frequencies $(0.5,1.0$, and $2.0 \mathrm{~Hz})$ were manipulated through the programmable control circuit and EMVs. For the magnitude of compressive loading, it was modulated by tuning the magnitude of the pneumatic pressure exerted in the pneumatic chamber (Figure 1(a)) through an air pressure regulator (Figure 1(b)). It was expressed as 


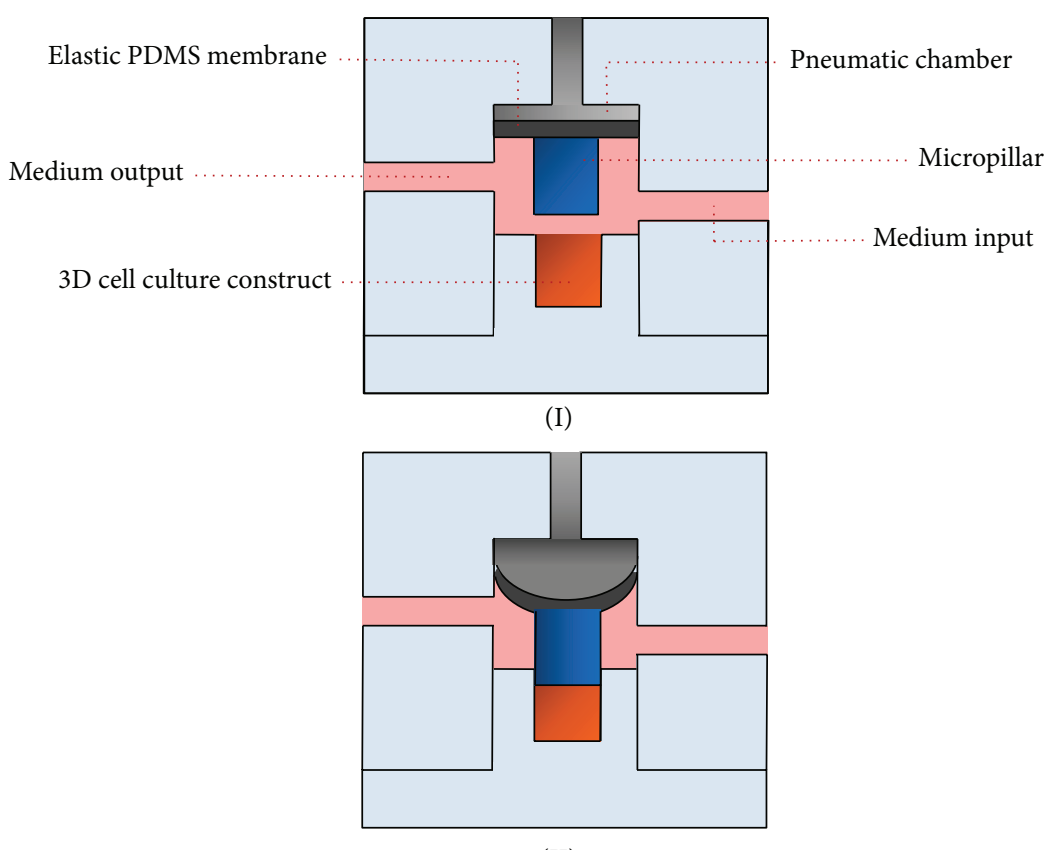

(II)

(a)

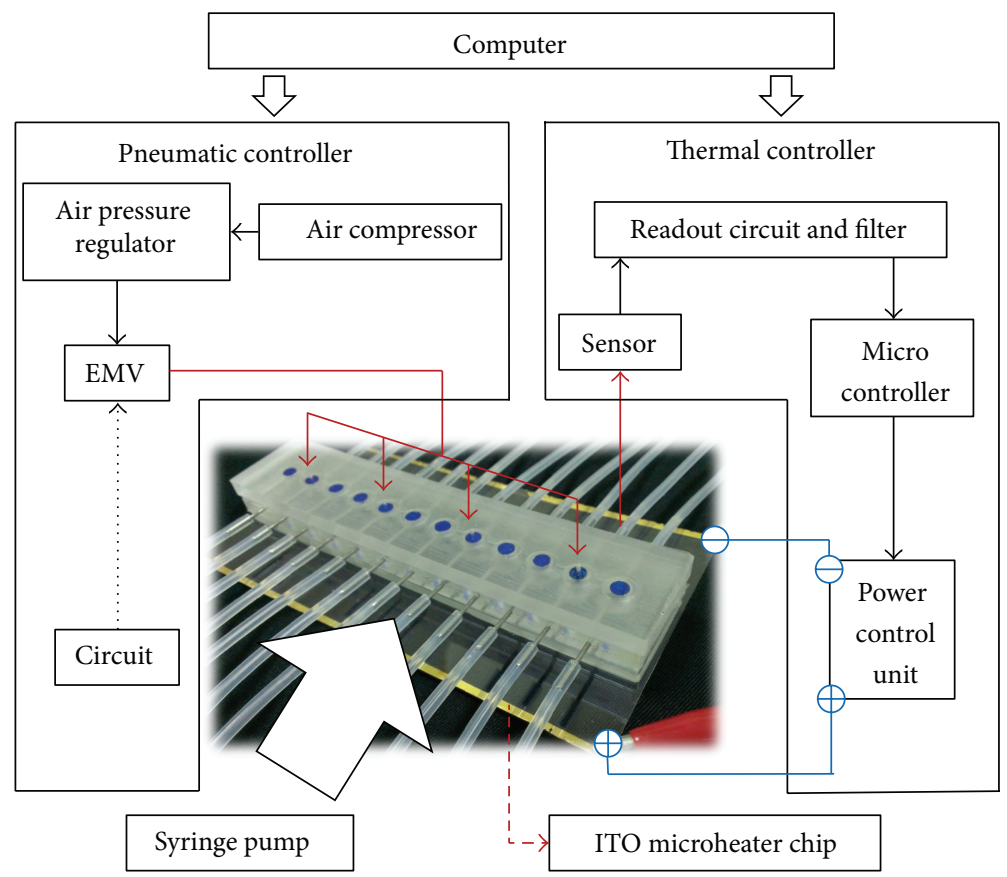

(b)

FIGURE 1: (a) Schematic illustration of the pneumatically-driven compressive loading mechanism: (I) the rest state and (II) the pneumaticallydriven deformation of an elastic PDMS membrane leading to the vertical movement of the connected micropillar that in turn generates compressive loading onto the 3D cell culture construct below (cross-sectional view) (a video clip is provided as a Supplementary Material available online at http://dx.doi.org/10.1155/2014/762570), and (b) the photograph of the microcell culture system, and the schematic illustration of overall experimental setup (EMV: electromagnetic valves; ITO: indium tin oxide).

compressive strain (the ratio of compressive deformation per unit length along the compressive axis) and quantified by the method reported previously [11]. Briefly, the dynamic deformation of a 3D cell culture construct was experimentally observed with the aid of a high-speed charge-coupled device (CCD) camera (MC1311, Mikrotron, Germany). In the measurements, the sequential action images of the dynamic deformation of a 3D cell culture construct were captured at the resolution speed of 100 frames $\mathrm{sec}^{-1}$. The compressive strain (\%) was then measured by the equation: 


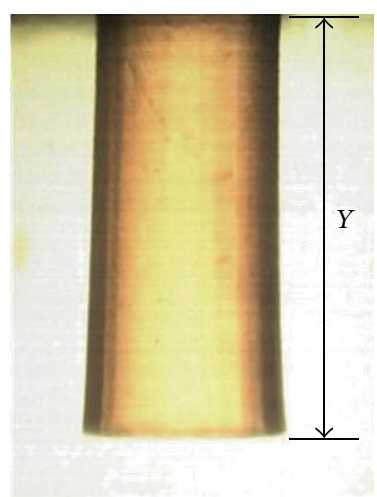

(I)

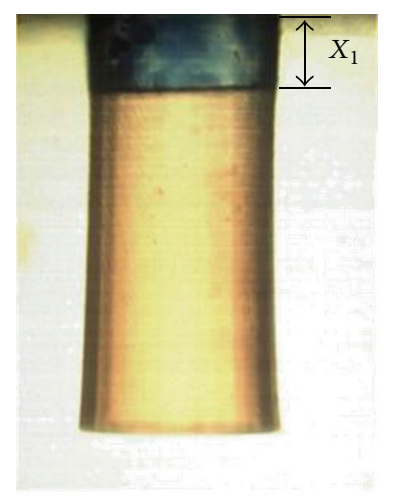

(II)

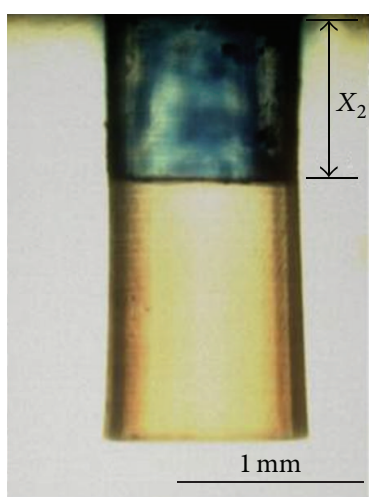

(III)

Strain (\%): $X / Y$ (II) $X_{1} / Y=19.6 \%$ strain

(III) $X_{2} / Y=40.4 \%$ strain

(a)

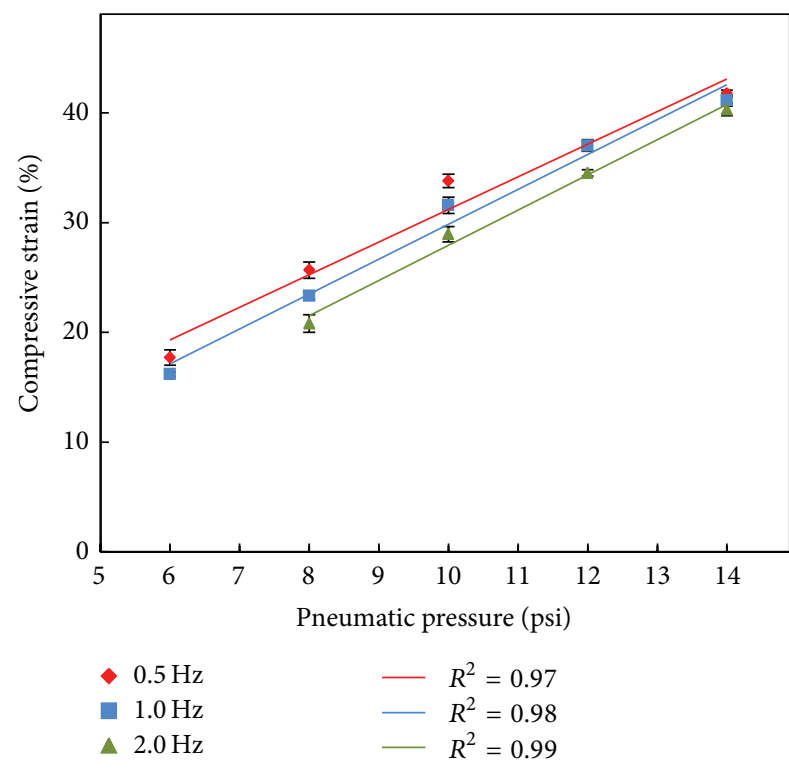

(b)

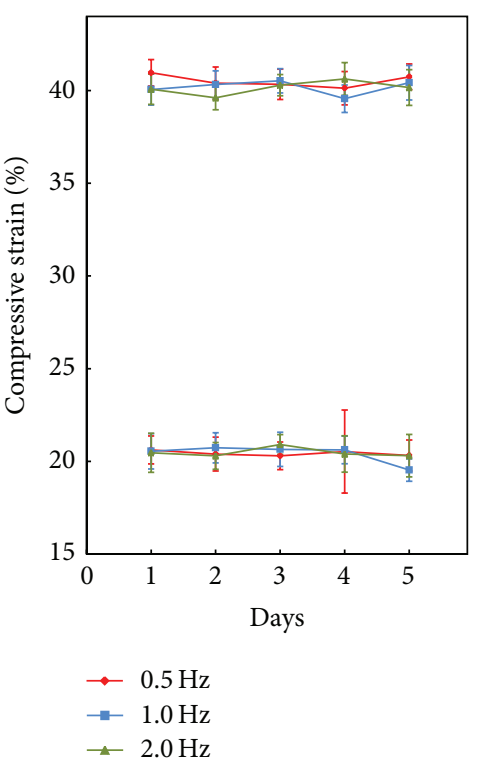

(c)

FIGURE 2: (a) Experimental quantification of the generated compressive strain by capturing the action images of micropillar using a high-speed CCD. The compressive strain (\%) is measured by the equation: $x$ (deformation length) $y$ (height of 3D cell culture construct; the photograph (I) $* 100 \%$. Photographs (II)-(III) showed the observed deformation lengths of $0.45 \mathrm{~mm}\left(X_{1}\right)$ and $0.93 \mathrm{~mm}\left(X_{2}\right)$ with the corresponding compressive strain of $19.6 \%$ and $40.4 \%$, respectively. (b) The quantitative relationship between the compressive strain and the pneumatic pressure applied under different operating frequency conditions $(0.5,1.0$, and $2.0 \mathrm{~Hz})$. (c) Evaluation of long-term stability of the generated compressive strain (lower and higher level: $20 \%$ and $40 \%$, resp.) under the operating frequencies of $0.5,1.0$, and $2.0 \mathrm{~Hz}$.

$x$ (the deformation length)/y (the height of $3 \mathrm{D}$ cell culture construct) $* 100 \%$. Figure 2 (a) showed the microscopic images of the $3 \mathrm{D}$ cell culture constructs subjected to the dynamic compressive loading with magnitudes of $20 \%$ and $40 \%$ strain. Based on above approaches, the quantitative relationships between the magnitude (6-14 psi) and the frequency $(0.5,1.0$, and $2.0 \mathrm{~Hz})$ of the pneumatic pressures exerted and the resulting compressive strain (\%) were experimentally determined. In this work, furthermore, the dynamic compressive stimulation was subjected to chondrocytesencapsulating 3D culture constructs for up to 5 days. In order to examine the long-term stability of dynamic compressive loading, the daily measurements of the compressive strain (20\% and 40\%) generated under different operating frequencies $(0.5,1.0$, and $2.0 \mathrm{~Hz})$ were carried out, based on the approach aforementioned.

\subsection{Perfusion 3D Articular Chondrocyte Culture under Differ-} ent Dynamic Compressive Loading. Primary articular chondrocytes were isolated from the femorotibial joint as previously described [3]. The cell suspension thus obtained 
was assessed microscopically for cell number and viability using $0.4 \%(\mathrm{w} / \mathrm{v})$ trypan blue in phosphate buffered saline (PBS; Invitrogen, Taiwan). Only cell preparations with cell viability greater than $95 \%$ were then used. In this study, the primary articular chondrocytes were encapsulated in alginate hydrogel to form a 3D cell culture construct. Briefly, the isolated cells were then immobilized in $6 \%(\mathrm{w} / \mathrm{v})$ alginate hydrogel at a cell density of $2.4 \times 10^{7}$ cells $\mathrm{mL}^{-1}$. The mixture was subsequently loaded into the cylindrical cavity in the microbioreactors (Figure 1(a)). After sample loading and device assembling, each microbioreactor was perfused with $0.5 \mathrm{M}$ calcium chloride solution at a flow rate of $1 \mu \mathrm{L} \mathrm{min}^{-1}$ for $25 \mathrm{~min}$ for the solidification of the alginate hydrogel. This was followed by perfusion articular chondrocyte culture under a serial compressive loading settings (strain: $20 \%$ and 40\%; operating frequency: $0.5,1.0$, and $2.0 \mathrm{~Hz}$; daily regimen: three consecutive cycles of 1 hour loading and 1 hour relaxation) for up to 5 days using the established experimental setup (Figure 1(b)). In this research, Dulbecco's Modified Eagle's Medium (DMEM) (with $1000 \mathrm{mg} \mathrm{L}^{-1}$ glucose, $25 \mathrm{mM}$ HEPES, without sodium bicarbonate; unless stated otherwise all chemicals were purchased from Sigma, Taiwan) supplemented with $10 \%$ foetal bovine serum (Invitrogen, Taiwan), $2 \%$ antibiotic/antimycotic solution, and $50 \mu \mathrm{g} \mathrm{mL}^{-1}$ ascorbic acid was continuously perfused to the individual microbioreactors at a flow rate of $60 \mu \mathrm{L} \mathrm{hr}^{-1}$. After a 5-day perfusion cell culture under compressive stimulation, the cell viability was observed microscopically through fluorescent staining. In addition, both culture medium and $3 \mathrm{D}$ cell culture constructs were collected to evaluate the proliferative, metabolic, and biosynthetic activities of articular chondrocytes by measuring the cellular DNA, lactic acid, GAGs, and hydroxyproline levels, respectively.

\subsection{Bioassays}

2.4.1. Evaluation of Cell Viability and Proliferation. The viability of the articular chondrocytes in alginate hydrogel under different compressive stimulation was evaluated using a fluorescent dye kit (LIVE/DEAD Viability/Cytotoxicity Kit L-3224, Molecular Probes). After cell culture, briefly, the remaining culture medium inside the microbioreactor chambers was removed. $10 \mu \mathrm{L}$ of the dye reagent containing $1 \mu \mathrm{M}$ calcein and $2 \mu \mathrm{M}$ ethidium homodimer-1, prepared according to the manufacturer's instruction, was loaded into each microbioreactor chamber. After $20 \mathrm{~min}$ incubation, the images of live (green) and dead (red) cells were captured using a confocal microscope (LSM 510 META, Zeiss, Germany). Cell viability was then quantified by counting the live (green) and dead (red) cells using a software program (SimplePCI version 5.2.1, Compix Inc., PA, USA) [19]. In addition, the cell proliferation of articular chondrocytes was evaluated by quantifying the DNA contents of cells. The DNA content of cells was detected according to Hoemann et al. [20]. Calf thymus DNA (5 to $125 \mathrm{ng}$ ) was used as standard.

2.4.2. Lactic Acid. The lactic acid produced and released into culture medium was measured using a Lactate Reagent Kit
(Trinity Biotech Plc., Ireland) [21]. The assay was carried out as directed by manufacturer's instructions. A lactate solution at a concentration of $50 \sim 500 \mathrm{mg} \mathrm{L}^{-1}$ made from dissolving lactate sodium salt in deionised water (DI) water was used as standard.

2.4.3. Glycosaminoglycans (GAGs) and Hydroxyproline. A colorimetric reaction based on Farndale et al. [22] was used to detect GAGs. The method used in this experiment was modified according to Hoemann et al. [20]. Absorbance at $540 \mathrm{~nm}$ was read using a microplate reader (Sunrise, Tecan Ltd, Taiwan) and chondroitin sulphate. A sodium salt in the range between $0.01 \mu \mathrm{g}$ and $1.25 \mu \mathrm{g}$ was used as standard. Moreover, total collagen production was assayed as hydroxyproline content after hydrolysis. The hydroxyproline content of insoluble collagen was determined according to Stegemann and Stalder [23]. In this evaluation, the hydroxyproline was assayed by a modified method by Urban and McMullin [24].

2.5. Statistical Analysis. Data from at least three separate experiments were analyzed and presented as mean \pm standard deviation. For a given experiment, each condition was tested in triplicate $(n=9)$. One-way ANOVA analysis with a statistical significance level of 0.05 was used to examine the effects of different compressive stimulation on the proliferative, metabolic, and biosynthetic activities of chondrocytes after 5 days of culture. The Tukey Honestly Significant Difference (HSD) post hoc test was used to compare the differences between two compressive loading conditions when the null hypothesis of ANOVA analysis was rejected.

\section{Results and Discussions}

3.1. Effect of Operating Conditions on the Generation of Dynamic Compressive Loading. In this study, a pneumatically-driven membrane-based actuation scheme was adopted to create compressive loading on $3 \mathrm{D}$ cell culture constructs (Figure 1(b)). This working mechanism has also been utilized in a wide variety of micropumps in various microfluidic systems $[25,26]$. Different from the other approaches to generate such mechanical movements, pneumatically-driven mechanism is generally regarded to have lower fabrication cost and simpler fabrication and operation process $[25,26]$. In the microcell culture system, the magnitude of the pneumatic pressure exerted in the pneumatic chamber (Figure 1(a)) and its operating frequency are two key parameters that can manipulate the dynamic compressive loading on a $3 \mathrm{D}$ cell culture construct. Figure 2(b) shows the quantitative relationships between the operating conditions and the resulting compressive loadings. For a given operating frequency, the generated compressive strain increased proportionally $\left(R^{2}: 0.97,0.98\right.$, and 0.99 for the $0.5,1.0$, and $2.0 \mathrm{~Hz}$, resp.) with the increase of the applied pneumatic pressure within the experimental conditions investigated (Figure 2(b)). Neglecting the mechanical resistance of $3 \mathrm{D}$ cell culture construct, the above finding is due to the fact that the resultant compressive strain is directly proportional to the deformation magnitude of PDMS 
membrane. The key moving component in the mechanism of compressive loading generation is the elastic PDMS membrane and its connected micropillar (Figure 1(a)). The pneumatically-driven deformation of a PDMS membrane is well described as follows [27]:

$$
w_{o}=\frac{p a^{2}}{4 \sigma_{o} h},
$$

where $w_{o}, p, a, \sigma_{o}$, and $h$ represent the maximum magnitude of membrane deformation, applied pneumatic pressure, radius of PDMS membrane, intrinsic tensile stress, and thickness of membrane, respectively.

From above equation, it can be observed that the deformation magnitude of a PDMS membrane is proportional to the magnitude of pneumatic pressure applied. These could reasonably explain the proportional relationship between the applied pneumatic pressure and the resultant compressive strain as observed in Figure 2(b). Moreover, it was also found that the generated compressive strain decreased with the increase of operating frequency (Figure 2(b)). At a given pneumatic pressure, the dynamic process to load a pneumatic chamber with an air pressure is time-dependent [28, 29]. Higher operating frequency implies shorter imposition time (e.g., 2.0, 1.0, and $0.5 \mathrm{sec}$ round $^{-1}$ for the $0.5,1.0$, and $2.0 \mathrm{~Hz}$, resp.) of pneumatic pressure input. This could accordingly lead to reduced PDMS membrane deformation and thus the lower level of compressive strain is generated. The experiments also found that the maximum frequency and strain that can be obtained in the system are $4 \mathrm{~Hz}$ and $50 \%$ stain, respectively. In the study, furthermore, a dynamic compressive stimulation was applied onto an articular chondrocytesencapsulating 3D culture construct for 5 days. For a more accurate investigation, the long-term stability of the compressive loading was experimentally examined. This issue has been generally ignored in several studies based on the same pneumatically-driven membrane-based actuations [25, 26, 28]. Figure 2(c) exhibited the long-term variations of the compressive strain generated under different operating conditions. For the lower compressive strain range of around $20 \%$, the measured compressive strains were found to have no significant difference $(P>0.05$, ANOVA) during the 5 days operation under a given operating frequency tested. Furthermore, when the compressive strain generated under different operating frequencies were compared, they were measured to have no statistical difference $(P>0.05$, ANOVA) at each time point investigated during the 5-day operation. Similar results were also found at the higher compressive strain range of about $40 \%$. Taken together, the above findings might indicate that the proposed mechanism for dynamic compressive loading generation was capable of providing a stable compressive loading during the cell culture period.

\subsection{Effect of Dynamic Compressive Loading on the Cell Viabil-} ity, Proliferation, and Metabolic Activities of Articular Chondrocytes. In this study, the primary articular chondrocytes cultured in $3 \mathrm{D}$ alginate hydrogel constructs were subjected to various compressive stimulation conditions (strain: $20 \%$, and $40 \%$; frequencies: $0.5,1.0$, and $2.0 \mathrm{~Hz}$ ) for up to 5 days. In addition, the dynamic compressive stimulation regimen was based on 3 consecutive cycles per day, (one cycle is defined as 1 hour of loading followed by 1 hour of relaxation), which was reported to be more physiologically relevant $[1,30,31]$. In this study, the impact of the compressive stimulation on the articular chondrocyte viability was observed using a fluorescent dye staining and quantified using an image analysis. Figure 3 showed the microscopic images of the articular chondrocytes treated with different compressive stimulation conditions, in which the green and red dots represent the live and dead cells, respectively. Overall, the articular chondrocytes cultured under dynamic compressive loading kept the average cell viability as high as 95\%, without statistical difference $(P>$ 0.05 , ANOVA) among the experimental conditions tested. These outcomes were consistent with the previous findings showing that the cell viability of articular chondrocytes is not mechanically affected by the compressive strain being lower than 51.5\% [11]. Articular cartilage is a tissue designed to withstand compression during joint movement, and thus the articular chondrocytes are normally subjected to a wide range of mechanical loadings in vivo. In normal physiological conditions, a human articular cartilage is subjected to the compressive strain ranging from $20 \%$ to $30 \%$ in vivo $[1,32,33]$. In this study, the higher compressive strain around $40 \%$ is regarded as the excessive loading of articular cartilage during vigorous exercise [33]. Taken together, the above findings could indicate that the articular chondrocytes might keep high cell viability under normal physiological condition or even under the excessive compressive strain as high as $40 \%-$ $50 \%$.

Furthermore, the DNA content of cells was measured as an indicator of articular chondrocytes proliferation after 5 days of cell culture in this study. As shown in Figure 4(a), there was no statistical difference $(P>0.05$, ANOVA) between the control (no compressive stimulation) and the mechanical compression-treated cases, meaning that the stimulating frequency $(0.5,1.0$, and $2.0 \mathrm{~Hz})$ and the magnitude $(20 \%$, and $40 \%$ strain) of compressive stimulation play no role in the proliferation of articular chondrocytes within the experimental conditions investigated. The articular chondrocytes, accounting for less than $5 \%$ of tissue volume, are the only cell species existing in articular cartilage. It has been reported that articular chondrocytes could not significantly proliferate when they are cultured in 3D culture environment, whereas their proliferative activity might increase while cultured in 2D monolayer format [34]. The phenomenon of no positive effect on articular chondrocyte proliferation found in this study is also discovered in several primary articular chondrocyte $3 \mathrm{D}$ cultures no matter if they are treated with mechanical stimulation $[8,9]$ or not $[16]$.

In addition, the metabolic activities of articular chondrocytes treated with various ranges of compressive loading were evaluated by measuring the lactic acid production. Regarding articular chondrocytes metabolism, anaerobic glycolysis is the major metabolic pathway [35], from which the lactic acid is the main metabolic product. Therefore, the production of lactic acid was utilized to indicate the chondrocytes metabolic activity in this study. Within the experimental conditions explored, results (Figure 4(b)) revealed that the stimulating 


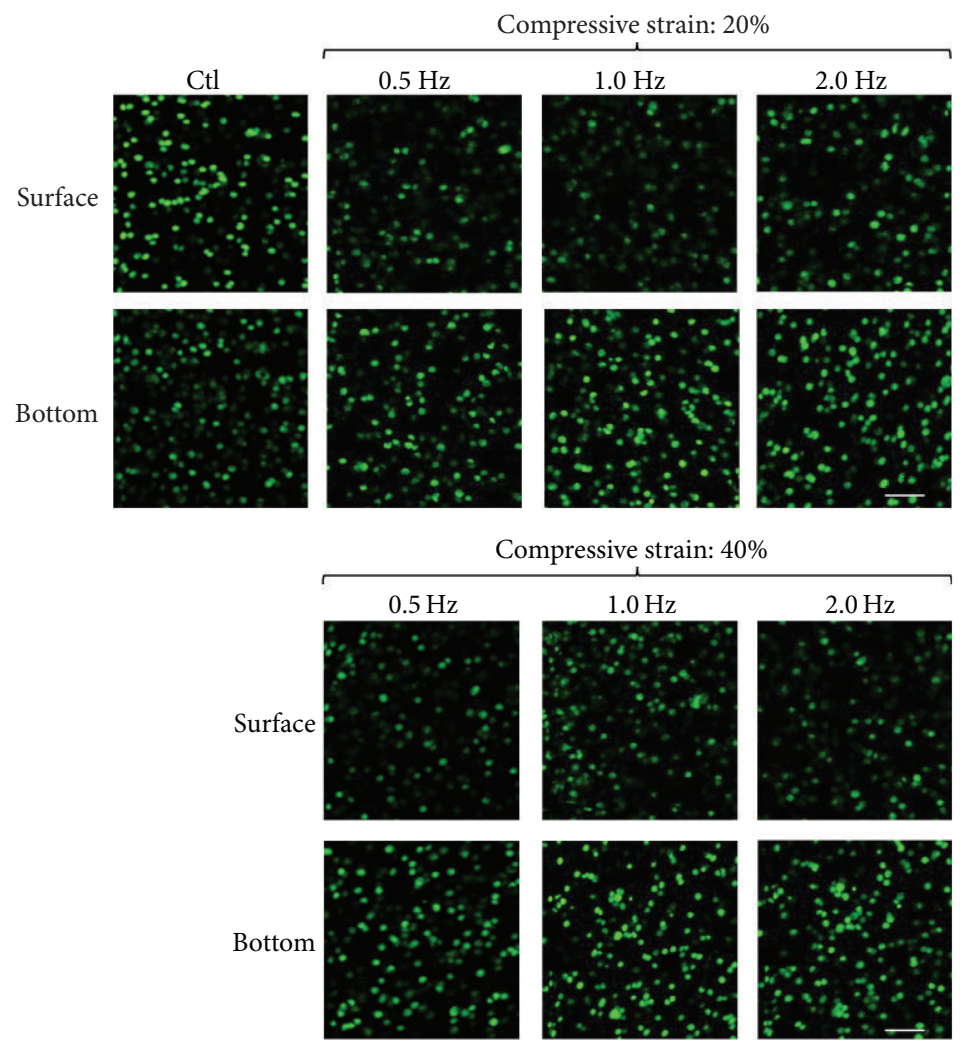

FIGURE 3: Microscopic observation of cell viability after 5 days of cell culture under different compressive stimulation conditions (strain magnitudes: $20 \%$ and $40 \%$; stimulating frequencies: $0.5,1.0$, and $2.0 \mathrm{~Hz}$ ) using the live/dead fluorescent dye. Green and red dots represent live and dead cells, respectively. The upper and lower images show the cells at the surface and the bottom of the 3D cell culture constructs, respectively.

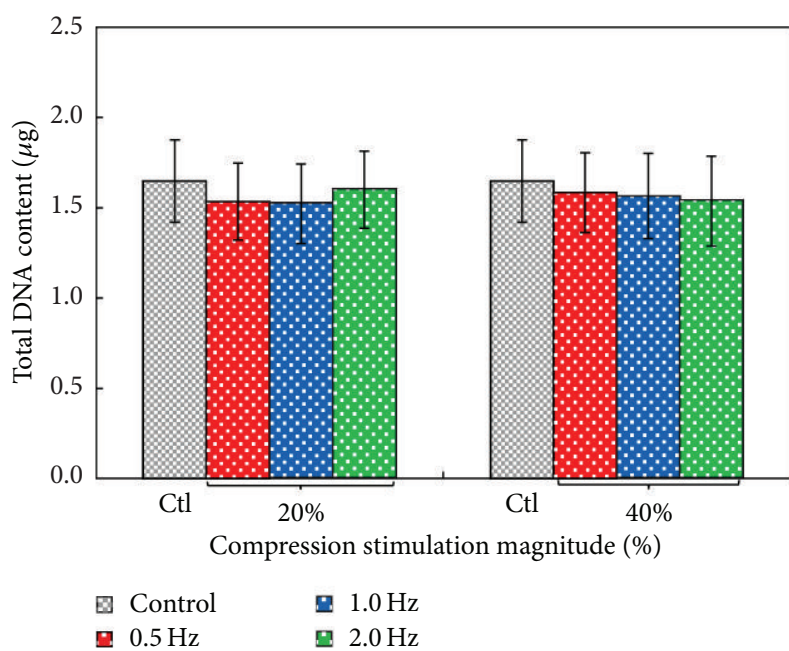

(a)

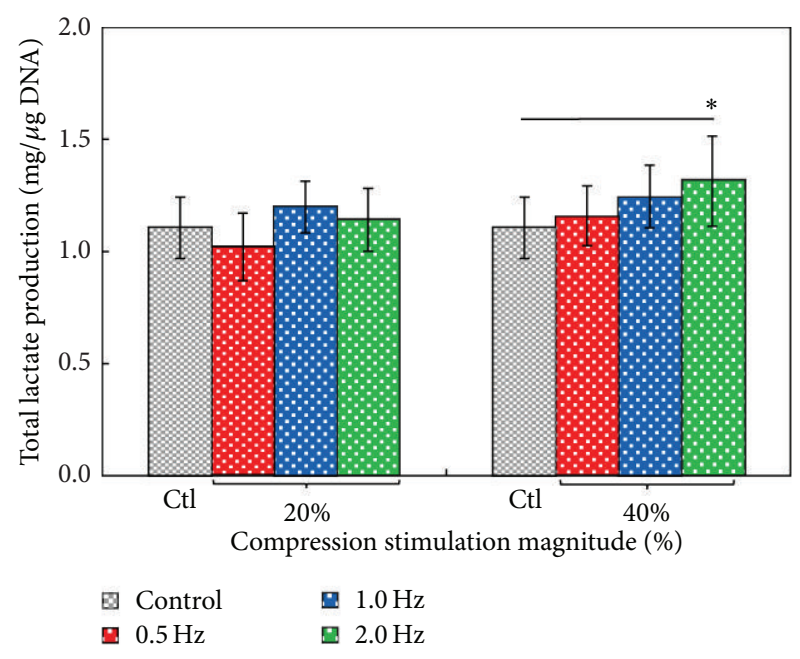

(b)

FIGURE 4: The evaluation of the (a) proliferative (DNA content measurement) and (b) metabolic (lactate production measurement) activities of the primary articular chondrocytes under different dynamic compressive loading conditions (as indicated) after 5 days of culture. The results are displayed as mean \pm standard deviation of 3 separate experiments $(n=9)$. Significant differences are expressed as ${ }^{*}(P<0.05)$. 


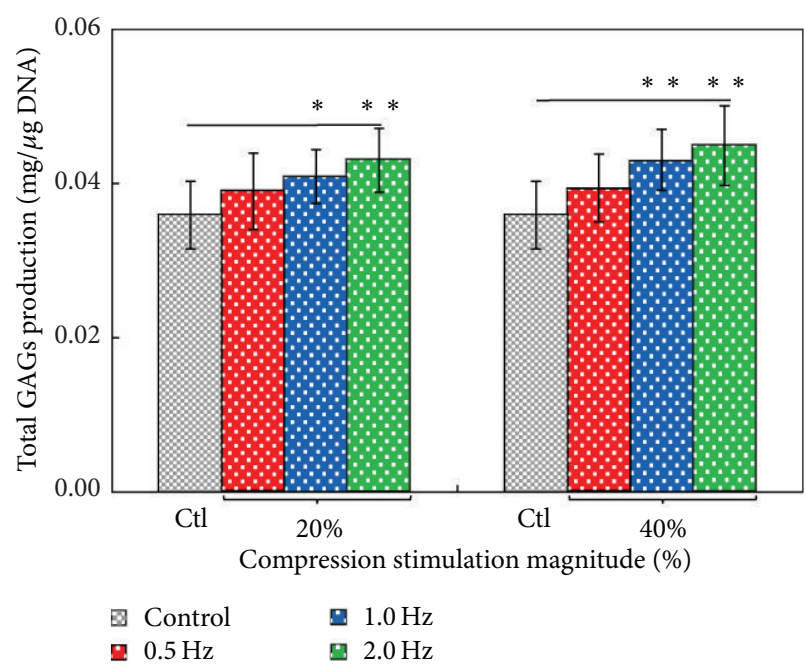

(a)

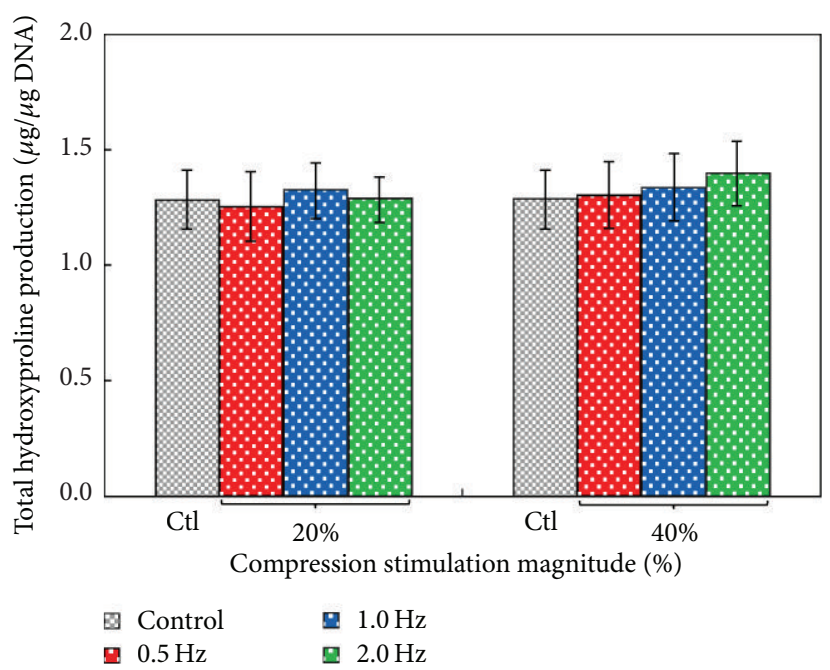

(b)

FIGURE 5: The evaluation of the biosynthetic activities ((a) GAGs and (b) hydroxyproline production) of the primary articular chondrocytes under different compressive loading conditions (as indicated) after 5 days of culture. The results are displayed as mean \pm standard deviation of 3 separate experiments $(n=9)$. Significant differences are expressed as ${ }^{*}(P<0.05)$ and ${ }^{* *}(P<0.01)$.

frequency of compressive loading did not significantly affect the metabolic activities of chondrocytes under the strain magnitude of $20 \%$. At the higher strain magnitude of $40 \%$, however, the frequency of compressive stimulation did have significant impact on the metabolic activities of articular chondrocytes $(P<0.05$, ANOVA $)$. The total lactic acid production of chondrocytes cultured under the stimulating frequency of $2 \mathrm{~Hz}$ ( $40 \%$ strain) was $18.77 \%$ statistically higher than that of the control case $(P<0.05)$. Nevertheless, the lactic acid production was not statistically different among the cells experiencing stimulating frequency lower than $2 \mathrm{~Hz}$. The dynamic compression at the $40 \%$ strain and $2 \mathrm{~Hz}$ is close to the compressive condition that human articular cartilage experiences during high intense exercise $[33,36]$. The finding disclosed in this study could indicate that the high frequency vigorous exercise might upregulate the metabolism of articular chondrocytes in comparison with the case without compressive stimulation.

\subsection{Effect of Dynamic Compressive Loading on the Biosyn-} thetic Activities of Articular Chondrocytes. Under compressive stimulation, several in vitro investigations revealed that the articular chondrocytes could rearrange their synthesis of ECM components [1,5-11]. However, few of them have reported the role of stimulating frequency on the biosynthetic activities of articular chondrocytes $[5,6,9]$. In this study, results (Figure 5(a)) exhibited that the stimulating frequency could significantly influence the GAGs synthesis of articular chondrocytes $(P<0.05$, ANOVA $)$ both at the two strain magnitudes (20\% and $40 \%)$ explored. In the ECM of articular cartilage, the GAGs are mainly responsible for the compressive stiffness of such tissue [10, 37]. It is reported that articular chondrocytes may synthesize more GAGs in responding to dynamic compressive loading [38]. For the two compressive strain groups tested, the total GAGs synthesis of articular chondrocytes cultured under the stimulating frequencies of $1 \mathrm{~Hz}$, and $2 \mathrm{~Hz}$ were statistically $(P<0.05$ for $1 \mathrm{~Hz}(20 \%$ strain $)$ and $P<0.01$ for $2 \mathrm{~Hz}(20 \%$ strain $)$ and 1 and $2 \mathrm{~Hz}$ (40\% strain)) higher than that of the control case. Nevertheless, such phenomenon was not found among the cells experiencing the stimulating frequency lower than $0.5 \mathrm{~Hz}$. The stimulating frequencies of $1 \mathrm{~Hz}$ and $2 \mathrm{~Hz}$ are about the frequency range of compressive loading that the human articular cartilages are subjected to during walking [39] and running [36] conditions, respectively. Therefore, the findings revealed in Figure 5(a) might imply that the GAGs biosynthesis of articular chondrocytes might be upregulated under these physiological conditions. In terms of collagen biosynthesis of chondrocytes, results (Figure 5(b)) demonstrated that the formats of compressive loading including stimulating magnitude and frequency did not significantly $(P>0.05$, ANOVA $)$ influence the total hydroxyproline production within the experimental conditions investigated.

As a whole, the above findings demonstrated that the GAGs synthesis was mechanically upregulated while the collagen production was not affected when $3 \mathrm{D}$ cultured chondrocytes were subjected to dynamic compressive stimulation. Reports in literature have also revealed similar research outcomes. For example, some studies have disclosed that dynamic compressive or tensile loading might increase the GAGs biosynthesis of articular chondrocytes but these mechanical stimulations were found to have no positive effect on the synthesis of collagen $[5,8,11,21,38]$. Nevertheless, other studies have exhibited different results $[1,6,31,37]$. For example, some studies have reported that dynamic compression has advantageous effect both on the production of GAGs and collagen $[1,6,31]$. Moreover, Hunter et al. [37] disclosed the adverse effect of dynamic compressive stimulation on GAGs and collagen production 
of articular chondrocytes. The reasons behind the above discrepancies are complicated. It is well accepted that the regimen of compressive stimulation [31], as well as the scaffolding material $[9,37]$, can influence the ECM synthesis of articular chondrocytes. In this study, the daily regimen of dynamic compressive stimulation was based on 3 consecutive cycles of 1 hour loading and 1 hour relaxation, which was reported to be more physiologically relevant $[1,30,31]$. In the investigations, moreover, the primary articular chondrocytes were microencapsulated in an alginate construct. In addition to its excellent biocompatibility [40-42], alginate hydrogel is a commonly used scaffolding material for cartilage tissue engineering because the phenotypic or functional stability of articular chondrocytes in this material can be maintained [43-45]. Furthermore, mammalian cells are sensitive to extracellular microenvironments [16]. To precisely study the cellular responses to extracellular conditions, a stable and homogenous culture environment is crucial because it can provide a well-defined and quantifiable culture condition. Unlike the most of cell culture models (e.g., the static or larger scale cell culture devices $[1,5-7,9,10,13])$ adopted for the similar investigations, one of the technical highlights in this study is the use of a perfusion-based microscale cell culture platform capable of providing dynamic compressive stimulation to cells. It enables scientists to create more stable and well-controlled culture environments due to the continuous nutrient supply and waste removal [14, 17] and the phenomenon of low chemical gradients existing in 3D cell culture construct [14], respectively. As a whole, this study has utilized the microcell culture system to mainly explore the stimulating frequency effect of compressive loading on the articular chondrocytes functions. The use of the microcell culture system for more systematic researches will be required to reconcile the differences with data acquired through conventional methods.

\section{Conclusions}

In this study, the stimulating frequency $(0.5,1.0$, and $2.0 \mathrm{~Hz})$ effect of compressive loading (strain: 20 and $40 \%$ ) on the cell viability and proliferation as well as the metabolic and biosynthetic activities of articular chondrocytes were investigated. It was found that the dynamic compressive loadings explored in this study did not have significant impact on the articular chondrocyte viability and proliferation, in which the cells kept the cell viability as high as $95 \%$. As to the metabolic activity of articular chondrocytes, the frequency of compressive stimulation did have significant influence when the compressive strain was increased to $40 \%$, in which the lactic acid production of cells treated with the stimulating frequency of $2 \mathrm{~Hz}(40 \%$ strain) was $18.77 \%$ statistically higher than that of the case without mechanical stimulation. This compressive loading is close to the condition that human articular cartilage experiences during high intense exercise. For the biosynthetic activities of articular chondrocytes, results showed that the GAGs synthesis was mechanically upregulated when the stimulating frequency of compressive loading was set at $1 \mathrm{~Hz}$ and $2 \mathrm{~Hz}$. These are about the frequency range of compressive loading that the human articular cartilages subjected to during walking and running conditions, respectively. Nevertheless, the formats of compressive loading including stimulating magnitude and frequency did not have significant impact on the collagen production of articular chondrocytes within the experimental conditions explored. As a whole, the research findings above are found fundamentally important both for articular cartilage tissue engineering and articular cartilage rehabilitation.

\section{Conflict of Interests}

The authors declare that there is no conflict of interests regarding the publication of this paper.

\section{Authors' Contribution}

Wan-Ying Lin, Yu-Han Chang, Hsin-Yao Wang, Tzu-Chi Yang and Tzu-Keng Chiu contributed equally to this work.

\section{Acknowledgment}

The authors would like to thank the Chang Gung Memorial Hospital (CMRPD1C0121) for financial support.

\section{References}

[1] R. L. Mauck, M. A. Soltz, C. C. B. Wang et al., "Functional tissue engineering of articular cartilage through dynamic loading of chondrocyte-seeded agarose gels," Journal of Biomechanical Engineering, vol. 122, no. 3, pp. 252-260, 2000.

[2] L. A. McMahon, P. J. O'Brien, and P. J. Prendergast, "Biomechanics and mechanobiology in osteochondral tissues," Regenerative Medicine, vol. 3, no. 5, pp. 743-759, 2008.

[3] M. Brittberg, E. Sjögren-Jansson, M. Thornemo et al., "Clonal growth of human articular cartilage and the functional role of the periosteum in chondrogenesis," Osteoarthritis and Cartilage, vol. 13, no. 2, pp. 146-153, 2005.

[4] T. M. Griffin and F. Guilak, "The role of mechanical loading in the onset and progression of osteoarthritis," Exercise and Sport Sciences Reviews, vol. 33, no. 4, pp. 195-200, 2005.

[5] D. A. Lee and D. L. Bader, "Compressive strains at physiological frequencies influence the metabolism of chondrocytes seeded in agarose," Journal of Orthopaedic Research, vol. 15, no. 2, pp. 181-188, 1997.

[6] T. Davisson, S. Kunig, A. Chen, R. Sah, and A. Ratcliffe, "Static and dynamic compression modulate matrix metabolism in tissue engineered cartilage," Journal of Orthopaedic Research, vol. 20, no. 4, pp. 842-848, 2002.

[7] C. R. Lee, A. J. Grodzinsky, and M. Spector, "Biosynthetic response of passaged chondrocytes in a type II collagen scaffold to mechanical compression," Journal of Biomedical Materials Research A, vol. 64, no. 3, pp. 560-569, 2003.

[8] O. Schmidt, J. Mizrahi, J. Elisseeff, and D. Seliktar, "Immobilized fibrinogen in PEG hydrogels does not improve chondrocytemediated matrix deposition in response to mechanical stimulation," Biotechnology and Bioengineering, vol. 95, no. 6, pp. 10611069, 2006. 
[9] I. Villanueva, D. S. Hauschulz, D. Mejic, and S. J. Bryant, "Static and dynamic compressive strains influence nitric oxide production and chondrocyte bioactivity when encapsulated in PEG hydrogels of different crosslinking densities," Osteoarthritis and Cartilage, vol. 16, no. 8, pp. 909-918, 2008.

[10] O. Preiss-Bloom, J. Mizrahi, J. Elisseeff, and D. Seliktar, "Realtime monitoring of force response measured in mechanically stimulated tissue-engineered cartilage," Artificial Organs, vol. 33, no. 4, pp. 318-327, 2009.

[11] M. H. Wu, H. Y. Wang, C. L. Tai et al., "Development of perfusion-based microbioreactor platform capable of providing tunable dynamic compressive loading to 3-D cell culture construct-Demonstration study of the effect of compressive stimulation on articular chondrocyte functions," Sensors and Actuators B: Chemical, vol. 176, pp. 86-96, 2013.

[12] A. Hasan, K. Ragaert, W. Swieszkowski et al., "Biomechanical properties of native and tissue engineered heart valve constructs," Journal of Biomechanics, 2013.

[13] M. M. G. Grafton, L. Wang, P.-A. Vidi, J. Leary, and S. A. Lelièvre, "Breast on-a-chip: mimicry of the channeling system of the breast for development of theranostics," Integrative Biology, vol. 3, no. 4, pp. 451-459, 2011.

[14] M.-H. Wu, J. P. G. Urban, Z. Cui, and Z. F. Cui, "Development of PDMS microbioreactor with well-defined and homogenous culture environment for chondrocyte 3-D culture," Biomedical Microdevices, vol. 8, no. 4, pp. 331-340, 2006.

[15] M.-H. Wu, J. P. G. Urban, F. C. Zhan, Z. Cui, and X. Xu, "Effect of extracellular $\mathrm{pH}$ on matrix synthesis by chondrocytes in $3 \mathrm{D}$ agarose gel," Biotechnology Progress, vol. 23, no. 2, pp. 430-434, 2007.

[16] A. Hasan, A. Memic, N. Annabi et al., "Electrospun scaffolds for tissue engineering of vascular grafts," Acta Biomaterialia, vol. 10, no. 1, pp. 11-25, 2014.

[17] P. M. Van Midwoud, E. Verpoorte, and G. M. M. Groothuis, "Microfluidic devices for in vitro studies on liver drug metabolism and toxicity," Integrative Biology, vol. 3, no. 5, pp. 509-521, 2011.

[18] J.-L. Lin, M.-H. Wu, C.-Y. Kuo, K.-D. Lee, and Y.-L. Shen, "Application of indium tin oxide (ITO)-based microheater chip with uniform thermal distribution for perfusion cell culture outside a cell incubator," Biomedical Microdevices, vol. 12, no. 3, pp. 389-398, 2010.

[19] M.-H. Wu, S.-B. Huang, Z. Cui, Z. Cui, and G.-B. Lee, "A high throughput perfusion-based microbioreactor platform integrated with pneumatic micropumps for three-dimensional cell culture," Biomedical Microdevices, vol. 10, no. 2, pp. 309-319, 2008.

[20] C. D. Hoemann, J. Sun, V. Chrzanowski, and M. D. Buschmann, "A multivalent assay to detect glycosaminoglycan, protein, collagen, RNA, and DNA content in milligram samples of cartilage or hydrogel-based repair cartilage," Analytical Biochemistry, vol. 300, no. 1, pp. 1-10, 2002.

[21] M.-H. Wu, H.-Y. Wang, H.-L. Liu et al., "Development of highthroughput perfusion-based microbioreactor platform capable of providing tunable dynamic tensile loading to cells and its application for the study of bovine articular chondrocytes," Biomedical Microdevices, vol. 13, no. 4, pp. 789-798, 2011.

[22] R. W. Farndale, D. J. Buttle, and A. J. Barrett, "Improved quantitation and discrimination of sulphate glycosaminoglycans by use of dimethylmethylene blue," Biochimica et Biophysica Acta, vol. 883 , no. 2 , pp. $173-177,1986$.
[23] H. Stegemann and K. Stalder, "Determination of hydroxyproline," Clinica Chimica Acta, vol. 18, no. 2, pp. 267-273, 1967.

[24] J. P. G. Urban and J. F. McMullin, "Swelling pressure of the intervertebral disc: influence of proteoglycan and collagen contents," Biorheology, vol. 22, no. 2, pp. 145-157, 1985.

[25] M.-H. Wu, S.-B. Huang, Z. Cui, Z. Cui, and G.-B. Lee, "Development of perfusion-based micro 3-D cell culture platform and its application for high throughput drug testing," Sensors and Actuators B: Chemical, vol. 129, no. 1, pp. 231-240, 2008.

[26] W.-B. Lee, Y.-H. Chen, H.-I. Lin, S.-C. Shiesh, and G.-B. Lee, "An integrated microfluidic system for fast, automatic detection of C-reactive protein," Sensors and Actuators B: Chemical, vol. 157, no. 2, pp. 710-721, 2011.

[27] R. Schellin, G. Hess, W. Kühnel et al., "Measurements of the mechanical behaviour of micromachined silicon and siliconnitride membranes for microphones, pressure sensors and gas flow meters," Sensors and Actuators A: Physical, vol. 41, no. 1-3, pp. 287-292, 1994.

[28] S.-B. Huang, M.-H. Wu, Z. Cui, Z. Cui, and G.-B. Lee, "A membrane-based serpentine-shape pneumatic micropump with pumping performance modulated by fluidic resistance," Journal of Micromechanics and Microengineering, vol. 18, no. 4, Article ID 045008, 2008.

[29] S. B. Huang, S. S. Wang, C. H. Hsieh, Y. C. Lin, C. S. Lai, and M. H. Wu, "An integrated microfluidic cell culture system for high-throughput perfusion three-dimensional cell culturebased assays: effect of cell culture model on the results of chemosensitivity assays," Lab on a Chip, vol. 13, no. 6, pp. 11331143, 2013.

[30] C. T. Hung, R. L. Mauck, C. C.-B. Wang, E. G. Lima, and G. A. Ateshian, "A paradigm for functional tissue engineering of articular cartilage via applied physiologic deformational loading," Annals of Biomedical Engineering, vol. 32, no. 1, pp. 3549, 2004.

[31] K. W. Ng, R. L. Mauck, C. C.-B. Wang et al., "Duty cycle of deformational loading influences the growth of engineered articular cartilage," Cellular and Molecular Bioengineering, vol. 2, no. 3, pp. 386-394, 2009.

[32] C. Herberhold, S. Faber, T. Stammberger et al., "In situ measurement of articular cartilage deformation in intact femoropatellar joints under static loading," Journal of Biomechanics, vol. 32, no. 12, pp. 1287-1295, 1999.

[33] J. T. Bingham, R. Papannagari, S. K. Van de velde et al., "In vivo cartilage contact deformation in the healthy human tibiofemoral joint," Rheumatology, vol. 47, no. 11, pp. 1622-1627, 2008.

[34] J. Diaz-Romero, J. P. Gaillard, S. P. Grogan, D. Nesic, T. Trub, and P. Mainil-Varlet, "Immunophenotypic analysis of human articular chondrocytes: changes in surface markers associated with cell expansion in monolayer culture," Journal of Cellular Physiology, vol. 202, no. 3, pp. 731-742, 2005.

[35] R. B. Lee and J. P. G. Urban, "Evidence for a negative Pasteur effect in articular cartilage," Biochemical Journal, vol. 321, no. 1, pp. 95-102, 1997.

[36] P. G. Weyand, D. B. Sternlight, M. J. Bellizzi, and S. Wright, "Faster top running speeds are achieved with greater ground forces not more rapid leg movements," Journal of Applied Physiology, vol. 89, no. 5, pp. 1991-1999, 2000.

[37] C. J. Hunter, J. K. Mouw, and M. E. Levenston, "Dynamic compression of chondrocyte-seeded fibrin gels: effects on matrix accumulation and mechanical stiffness," Osteoarthritis and Cartilage, vol. 12, no. 2, pp. 117-130, 2004. 
[38] J. D. Kisiday, M. Jin, M. A. DiMicco, B. Kurz, and A. J. Grodzinsky, "Effects of dynamic compressive loading on chondrocyte biosynthesis in self-assembling peptide scaffolds," Journal of Biomechanics, vol. 37, no. 5, pp. 595-604, 2004.

[39] O. Gabay, D. J. Hall, F. Berenbaum, Y. Henrotin, and C. Sanchez, "Osteoarthritis and obesity: experimental models," Joint Bone Spine, vol. 75, no. 6, pp. 675-679, 2008.

[40] G. Klöck, A. Pfeffermann, C. Ryser et al., "Biocompatibility of mannuronic acid-rich alginates," Biomaterials, vol. 18, no. 10, pp. 707-713, 1997.

[41] C. L. Salgado, M. B. Oliveira, and J. F. Mano, "Combinatorial cell-3D biomaterials cytocompatibility screening for tissue engineering using bioinspired superhydrophobic substrates," Integrative Biology, vol. 4, no. 3, pp. 318-327, 2012.

[42] P. Dandoy, C. F. Meunier, C. Michiels, and B.-L. Su, "Hybrid shell engineering of animal cells for immune protections and regulation of drug delivery: towards the design of "Artificial Organs'”' PLoS ONE, vol. 6, no. 6, Article ID e20983, 2011.

[43] H. J. Häuselmann, R. J. Fernandas, S. S. Mok et al., "Phenotypic stability of bovine articular chondrocytes after long-term culture in alginate beads," Journal of Cell Science, vol. 107, no. 1, pp. 17-27, 1994.

[44] M. F. Amilton, R. H. Sarah, A. K. Tommy, M. K. Axel, F. Yngvar, and E. B. Jan, "Similar properties of chondrocytes from osteoarthritis joints and mesenchymal stem cells from healthy donors for tissue engineering of articular cartilage," PLoS ONE, vol. 8, no. 5, Article ID e62994, 2013.

[45] M. F. Rai, T. Graeve, S. Twardziok, and M. F. G. Schmidt, "Evidence for regulated interleukin-4 expression in chondrocytescaffolds under in vitro inflammatory conditions," PLoS ONE, vol. 6, no. 10, Article ID e25749, 2011. 


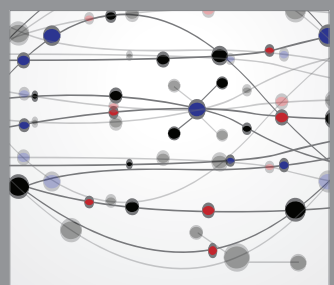

The Scientific World Journal
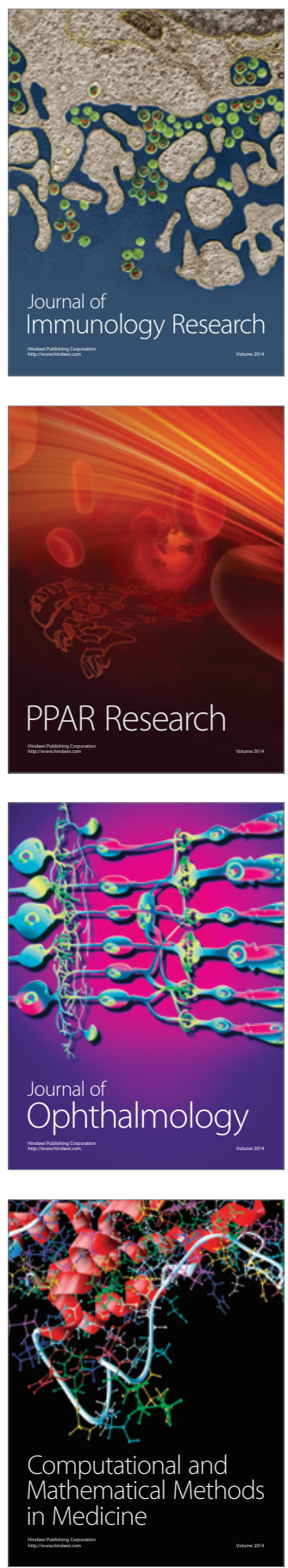

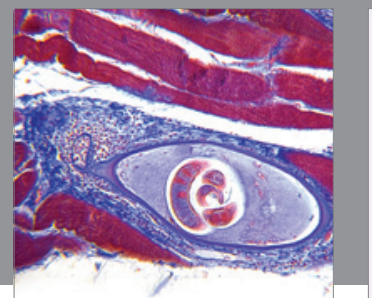

Gastroenterology

Research and Practice
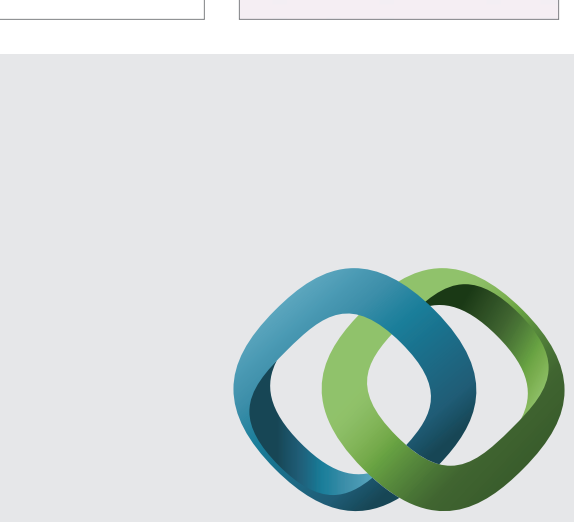

\section{Hindawi}

Submit your manuscripts at

http://www.hindawi.com
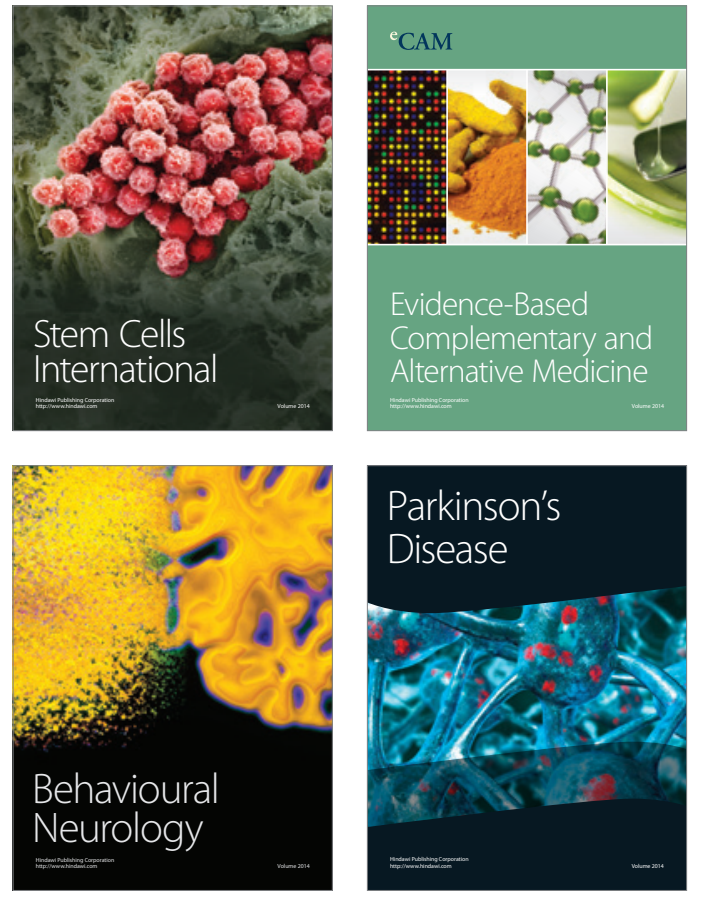
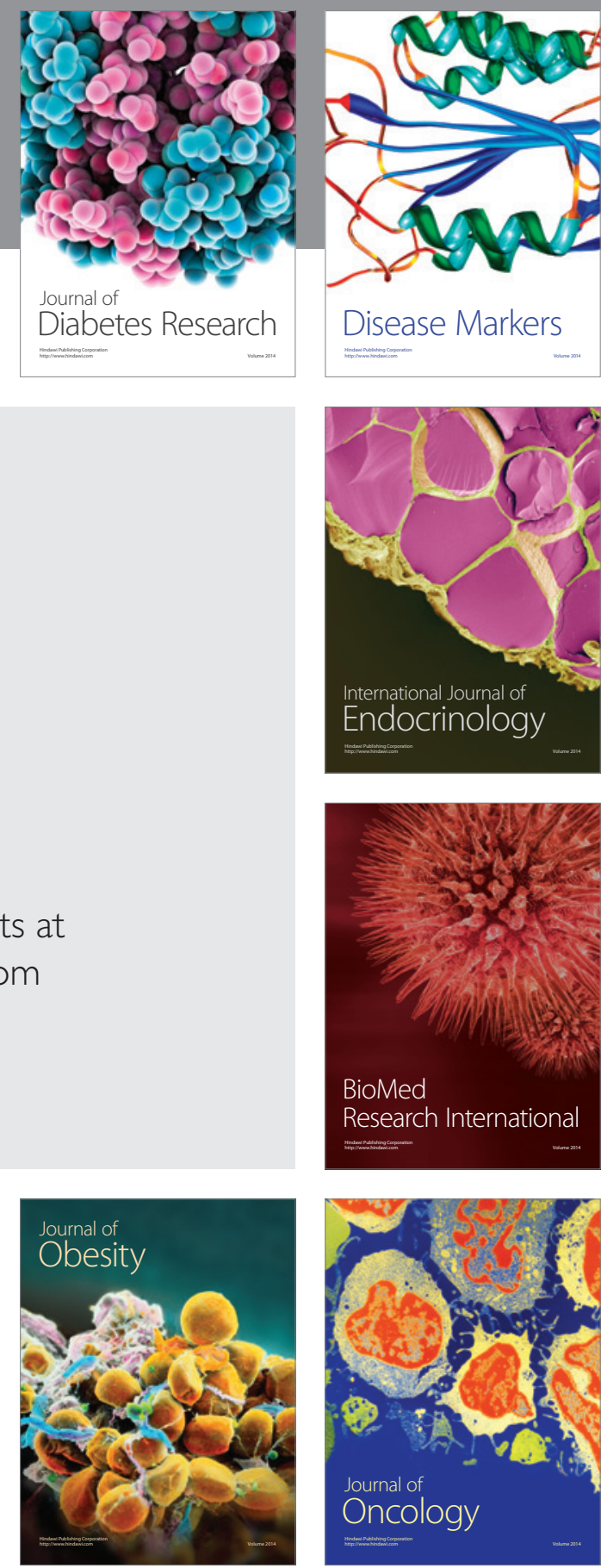

Disease Markers
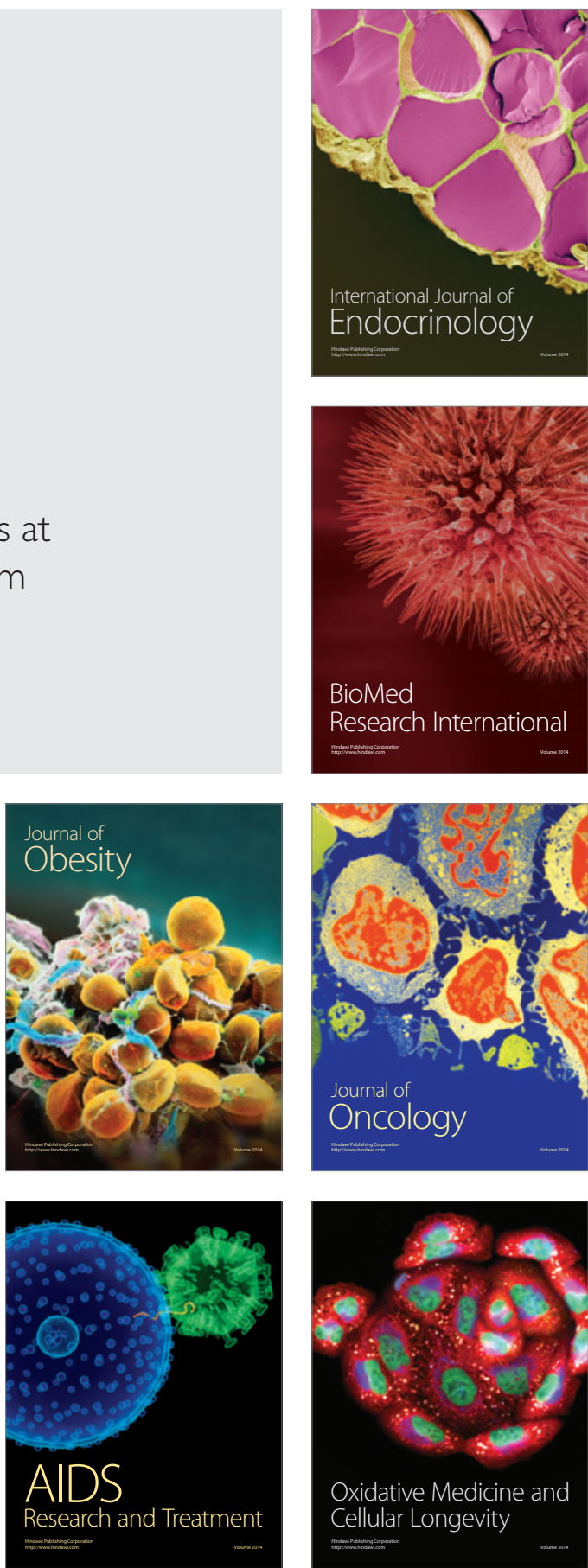